\section{Prevalencia de probable deterioro cognitivo en adultos mayores de una población mexicana utilizando el MMSE y el MoCA}

\section{Prevalence of probable cognitive impairment in older adults of a Mexican population using MMSE and MoCA}

José Miguel Sánchez-Nieto*

Víctor Manuel Mendoza-Núñez

Unidad de Investigación en Gerontología. Facultad de Estudios Superiores Zaragoza. Universidad Nacional Autónoma de México.

*Autor para correspondencia.

Correo electrónico: cheverego@hotmail.com (José Miguel Sánchez-Nieto).

Recibido el 13 de noviembre de 2019; aceptado el 24 de enero de 2020.

\section{RESUMEN}

Antecedentes: El Mini-Mental State Examination (MMSE) y el Montreal Cognitive Assessment (MoCA) son instrumentos de cribado usados frecuentemente para detectar probable deterioro cognitivo leve (DCL). El MoCA ha mostrado una mayor sensibilidad y especificidad en población clínica que el MMSE. Sin embargo,

es posible que en la comunidad se sobreestime la proporción de personas con DCL utilizando el MoCA. Por lo anterior, el objetivo es identificar en adultos mayores de la comunidad, en la ciudad de

México, la prevalencia de probable DCL utilizando el MoCA y el MMSE. Método: Se realizó un estudio descriptivo transversal, en una muestra de 231 participantes pertenecientes a grupos comunitarios con una edad entre 55 y 90 años y escolaridad de 0 a 22 años; se aplicó el MMSE y el MoCA. Resultados: Se obtiene una puntuación en MMSE de 25,0 $\pm 3,1$ y en MoCA de 19,8 $\pm 5,4$ con una correlación moderada $(r=0,59, p<0,05)$ entre ellos. Un $84 \%$ de la población utilizando el MoCA y un 23,8\% de la población utilizando el MMSE se clasifican con probable DCL. Conclusión: El MMSE puede dar una estimación más parecida a lo reportado en la literatura que el $\mathrm{MoCA}$ en la comunidad. Se discuten las implicaciones en políticas públicas, en el uso clínico y en investigación de una sobreestimación en la proporción de personas con probable DCL.

PALABRAS CLAVE: Deterioro cognitivo, problema de memoria, test de cribado.

\section{ABSTRACT}

Background: The Mini-Mental State Examination (MMSE) and the Montreal Cognitive Assessment (MoCA) are screening instruments frequently used to detect probable mild cognitive impairment (MCI). The MoCA has shown greater sensitivity and specificity in the clinical population than the MMSE. However, the proportion of people with MCI using the MoCA may be overestimated in the community. Therefore, the objective is to identify in older adults in the community in Mexico City the prevalence of probable DCL using the MoCA and the MMSE. Method: A cross-sectional descriptive study was carried out, in a sample of 231 participants belonging to community groups with an age between 55 and 90 years and schooling from 0 to 22 years; MMSE and MoCA were applied. Results: a score is obtained in MMSE of $25.0 \pm 3.1$ and in MoCA of $19.8 \pm 5.4$ with a moderate correlation $(r=0.59$, $p<0.05$ ) between them. $84 \%$ of the population using MoCA and $23.8 \%$ of the population using MMSE are classified as likely MCI. Conclusion: The MMSE can give an estimate more similar to what is reported in the literature than the MoCA in the community. The implications in public policies, in clinical use and in research of an overestimation in the proportion of people with probable MCI are discussed.

KEYWORDS: Cognitive Dysfunction, Memory Disorders, screening test.

\section{- INTRODUCCIÓN}

En las personas mayores, las pruebas cognitivas de cribado separan a quien tiene un envejecimiento cognitivo normal de aquellas con un probable trastorno neurológico como el Alzheimer, demencias vasculares o deterioro cognitivo leve (DCL), entre otros ${ }^{1}$. Las pruebas cognitivas de cribado, al ser de aplicación rápida y fácil, se utilizan para planear políticas públicas basadas en estudios epidemiológicos ${ }^{2}$; en la clínica, para realizar una intervención temprana ${ }^{3}$; y en la inves- tigación, para determinar factores de riesgo asociados con problemas cognitivos ${ }^{4}$.

Dos de los instrumentos de cribado más utilizados son el Mini-Mental State Examination (MMSE) y el Montreal Cognitive Assessment (MoCA). El MMSE fue publicado en 1975, para identificar el cambio cognitivo en pacientes psiquiátricos. Evalúa 11 dominios: orientación, registro, atención, cálculo, recuerdo, nominación, repetición, comprensión (verbal y escrita), escritura y construcción, y tiene una puntuación de 0 a 30. Se validó en diferentes poblaciones del mundo, tanto en 
clínicas de memoria como en comunidad, así como en diferentes padecimientos, entre ellos el Alzheimer, la demencia vascular y el DCL5. El MoCA es un instrumento diseñado para detectar DCL. Los dominios cognitivos que evalúa son memoria, lenguaje, funciones ejecutivas, habilidades visoespaciales, cálculo, abstracción, atención, concentración y orientación. Las tareas fueron elegidas con base en instrumentos neuropsicológicos sensibles para la detección de DCL, las cuales se adecuaron a lo largo de 5 años por medio de la práctica clínica. La puntuación es de 0 a 30. Se ha traducido a más de 50 idiomas. Las tareas del MoCA de memoria, funciones ejecutivas y habilidades visoespaciales son más complejas que en el MMSE6.

El MoCA tiene una mayor sensibilidad y especificidad que el MMSE para detectar probable DCL, así como otras patologías ${ }^{3,7,8}$. Sin embargo, el MMSE es de los instrumentos más utilizados en las ciencias de la salud ${ }^{9}$, por lo que clínicos e investigadores se encuentran más familiarizados con este instrumento, lo que facilita su aplicación e interpretación.

Ambos instrumentos tienen un rango de puntuación similar, de 0 a 30. Aunque el MoCA es más complejo que el MMSE, tiene un punto de corte mayor para detectar probable DCL. Mitchell, en una revisión sistemática sobre el MMSE, reporta que el punto de corte es $23^{5}$, mientras que Nasreddine et al. proponen en el MoCA un punto de corte de $26^{6}$. Este se ha encontrado en diferentes estudios ${ }^{10}$, incluso en población mexicana ${ }^{11}$.

La prevalencia del DCL en adultos mayores varía mucho dependiendo de su definición, del instrumento para su evaluación, así como la población estudiada. El rango en diferentes estudios se encuentra entre el $3 \%$ y el $53 \%{ }^{12}$. Es más probable que se encuentren falsos positivos en la población de comunidad en comparación con poblaciones en clínicas al utilizar el mismo punto de corte, por ejemplo, 26 en el MoCA; sin embargo, no se conoce en qué magnitud pueda generarse esta sobreestimación. Por lo anterior, el objetivo de este estudio es identificar en adultos mayores de la comunidad en la ciudad de México la prevalencia de probable DCL utilizando el MoCA y el MMSE.

\section{- MÉTODO}

Diseño: transversal descriptivo.

Participantes: la muestra estuvo integrada por 340 participantes pertenecientes a grupos comunitarios de la Ciudad de México. Se excluyó a 107 individuos por probable depresión, evaluada mediante el inventario de depresión de Yesavage ${ }^{12}$, y dos por tener epilepsia. La muestra final estuvo integrada por 231 participantes con una edad entre 55 y 90 años $(68 \pm 7,6)$, escolaridad de 0 a 22 años $(6,3 \pm 4)$, un $84 \%$ mujeres, y todos podían realizar actividades básicas e instrumentales de la vida diaria.

Instrumentos: se realizó una evaluación gerontológica integral en la que se incluyó el MMSE ${ }^{13}$ y el MoCA en su versión en español (http:// www.mocatest.org/pdf_files/test/MoCA-Test-Spanish.pdf).

Procedimiento: se acudió a diferentes centros de reunión de adultos mayores en la Ciudad de México, donde se invitó a participar a las personas mayores en un proyecto para prevenir y controlar las enfermedades crónicas en la vejez. Los participantes que aceptaron firmaron un consentimiento informado y fueron evaluados con el MMSE y el MoCA de forma individual por un profesional de la salud (psicólogos, enfermeras y médicos) previamente capacitados.

Análisis estadístico: para el análisis se utilizó el programa SPSS 20.0. Se obtuvo la media y la desviación estándar de las puntuaciones. Se realizó una correlación de Pearson entre las puntuaciones del MMSE y el MoCA. Para determinar un probable DCL utilizando el MoCA, se consideró un punto de corte de 26, se hizo la corrección de agregar un punto a las personas con menos de 12 años de escolaridad, de forma similar al estudio de Nasreddine et al. ${ }^{6}$. En el MMSE se utilizó el punto de corte de 23 propuesto por Mitchell5; no se realizó ninguna adaptación a la edad y a la escolaridad. También se formaron 6 grupos divididos por edad y escolaridad. Se compararon las puntuaciones del MoCA y del MMSE por grupo utilizando una $t$ de Student pareada considerando como significativa una $p<0,05$. Por último, se presentan los percentiles del MMSE y del MoCA.

\section{- RESULTADOS}

En el MMSE se encontró una media de 25 \$3,1 en un rango de 16 a 30 , con un sesgo de $-0,44$. Por otro lado, en el MoCA se encontró una media de $19,8 \pm 5,8$ en un rango de 6 a 30 con un sesgo de $-0,60$. Entre ambos instrumentos se encuentra una correlación de $r=0,57, p<0,01$. La prevalencia del DCL utilizando el punto de corte de 26 en el MoCA es del $83 \%$, mientras que empleando el punto de corte de 23 en el MMSE es del 23,8\%.

No hay diferencia significativa entre hombres y mujeres $(p>0,05)$; tampoco al comparar participantes con 0,3 o 5 ańos de escolaridad, por lo que se decidió formar un grupo con escolaridad en un rango de 0 a 5 años. La muestra se dividió en seis grupos considerando la edad ( 55 a 74 y 75 o más) y escolaridad ( 0 a 5 años, 6 a 9 años y de 10 o más años). Al realizar una comparación por grupos se encuentra que los participantes obtuvieron mayores puntuaciones en el MMSE que en el MoCA de forma significativa $(p<0,05)$ (tabla 1$)$.

Tomando en cuenta la edad y la escolaridad, los puntos de corte basados en el percentil 25 van de la puntuación 21 a la 26 en el MMSE, mientras que en el MoCA van del 8 al 21 (tabla 2). Considerando ese punto de corte, se encuentra una proporción del $20 \%$ con rendimiento cognitivo bajo.

Tabla 1. Comparación de la media y desviación estándar entre el MMSE y el MoCA considerando la edad y la escolaridad

\begin{tabular}{|c|c|c|}
\hline & MMSE & MoCA \\
\hline \multicolumn{3}{|l|}{ Edad de 55-74 años } \\
\hline Esc. de $0-5$ años ( $n=44)$ & $23,82 \pm 3,2$ & $16,27 \pm 5,0^{a}$ \\
\hline Esc. de 6-9 años ( $n=97)$ & $25,45 \pm 2,7$ & $21,34 \pm 4,1^{\mathrm{a}}$ \\
\hline Esc. $\geq 10$ años $(n=31)$ & $26,97 \pm 2,4$ & $23,55 \pm 3,1^{\mathrm{a}}$ \\
\hline \multicolumn{3}{|l|}{ Edad $\geq 75$ años } \\
\hline Esc. de 0-5 años ( $n=29)$ & $22,43 \pm 3,33$ & $15,2 \pm 5,7^{a}$ \\
\hline Esc. de 6-9 años (n=22) & $25,27 \pm 2,7$ & $19,6 \pm 4,6^{a}$ \\
\hline Esc. $\geq 10$ años $(n=8)$ & $27,75 \pm 1,8$ & $23,75 \pm 3,7^{a}$ \\
\hline
\end{tabular}

\section{- DISCUSIÓN}

El MoCA y el MMSE son dos instrumentos de cribado usados con frecuencia para detectar un probable DCL. Sin embargo, el uso inadecuado de los instrumentos puede ocasionar el sobreestimar la proporción de personas con un padecimiento cognitivo. Para determinar la magnitud de esta sobreestimación se identificó, en una población de la comunidad, la prevalencia de personas con probable DCL utilizando el MoCA y el MMSE. 
Tabla 2. Percentiles de las puntuaciones del MMSE y el MoCA considerando la edad y la escolaridad

\begin{tabular}{|l|c|c|c|c|c|c|c|}
\hline \multirow{2}{*}{ Edad } & \multirow{2}{*}{ Percentil } & \multicolumn{3}{|c|}{$\begin{array}{c}\text { MMSE } \\
\text { Escolaridad }\end{array}$} & \multicolumn{3}{c|}{$\begin{array}{c}\text { MoCA } \\
\text { Escolaridad }\end{array}$} \\
\cline { 3 - 8 } & & $0-5$ & $6-9$ & $\geq 10$ & $0-5$ & $6-9$ & $\geq 10$ \\
\hline \multirow{5}{*}{$55-74$} & 10 & 19 & 22 & 23 & 8 & 14 & 19 \\
\hline \multirow{5}{*}{5} & 21 & 23 & 25 & 11 & 18 & 21 \\
\hline & 50 & 24 & 26 & 28 & 16 & 22 & 24 \\
\hline & 75 & 26 & 27 & 29 & 21 & 24 & 26 \\
\hline \multirow{5}{*}{$\mathbf{7 5}$} & 90 & 29 & 29 & 29 & 24 & 26 & 29 \\
\hline & 10 & 17 & 22 & 25 & 7 & 13 & 20 \\
\hline & 25 & 20 & 23 & 26 & 11 & 16 & 20 \\
\hline & 50 & 22 & 25 & 27 & 13 & 20 & 22 \\
\hline & 75 & 24 & 28 & 30 & 19 & 24 & 27 \\
\hline
\end{tabular}

MMSE: Mini examen del estado mental; MoCA: evaluación cognitiva de Montreal.

Utilizando el punto de corte de 26 en la puntuación del MoCA, que es el recomendado por Nasreddine para detectar probable $\mathrm{DCL}^{6}$, encontramos una prevalencia del $84 \%$, mientras que empleando el punto de corte de 23, que es el recomendado por Mitchel $^{5}$, un 23,8\%. En varios estudios se halla una frecuencia como máximo del $53 \%{ }^{12}$, por lo que en el presente estudio se encuentra que con el de MoCA se excede la proporción de personas con DCL.

El sobreestimar la proporción de personas con probable DCL tiene repercusiones en políticas públicas, diagnóstico clínico y en investigación. En políticas públicas puede ocasionar que se desvíen recursos tanto económicos como de tiempo en personas que no tienen la enfermedad en lugar de utilizarlos para las personas que sí tienen un problema cognitivo, o su empleo en investigación para identificar tratamientos. En este sentido, la aplicación de los test de cribado a población general puede ocasionar una alta probabilidad de encontrar falsos positivos, como muestra nuestro estudio. Para evitar lo anterior, su aplicación debe restringirse en población con alta probabilidad de padecer un problema cognitivo; por ejemplo, en personas mayores de 80 ańos $^{14}$.

De manera clínica hay que considerar la utilidad del diagnóstico precoz de DCL. Este diagnóstico se ha propuesto como un estado previo a la demencia; sin embargo, solo aproximadamente un 50\% de las personas diagnosticadas con DCL progresan a demencia ${ }^{15}$. Además, continúa la necesidad de delinear y distinguir con mayor precisión las manifestaciones tempranas que permitan identificar una mayor probabilidad de progresión a demencia y desarrollar evidencia de una respuesta positiva a la intervención ${ }^{10,16}$.

Clínicamente, además de los instrumentos se realiza una entrevista para emitir un diagnóstico. Algunos de los elementos pertinentes a considerar al respecto son otras posibles enfermedades que pueden afectar a la cognición, como depresión, ansiedad o falta de vitamina $\mathrm{B}_{12}$; el valor agregado para el adulto mayor y su familia del diagnóstico, en particular si están adaptados a su vida; también probables repercusiones entre las que se encuentran estigmatización, reducción prematura de empleo y pérdida de apoyo para funciones cotidianas como conducir, permanecer independiente, tomar decisiones financieras, entre otras ${ }^{14}$.

En la investigación se utilizan con frecuencia ambos instrumentos para determinar si las personas presentan o no un problema cognitivo. Si se desea conocer los efectos de una intervención que mejore el DCL, probablemente se puede encontrar una mejoría, pero no debida a la intervención, sino a las características del padecimiento, al menos de lo que se conoce actualmente ${ }^{10,15,16}$.

Si se desea clasificar a las personas con DCL para determinar factores de riesgo o excluirlas de una investigación, utilizar el punto de corte de 26 en el MoCA puede dificultar identificar adultos mayores sanos; por ejemplo, en nuestro estudio únicamente el $17 \%$ no tendría DCL, además de agregar otras enfermedades comunes como depresión en el que en nuestro estudio se excluyó casi un tercio de la población inicial. En este caso, el MMSE da una estimación de la proporción de personas con DCL más cercana a lo encontrado en otros estudios ${ }^{12}$, por lo que sería más útil en estudios en la comunidad.

El MoCA tiene una alta correlación con el MMSE, por lo que puede ser utilizado también en investigación, pero es necesario realizar algunos ajustes, como un punto de corte diferente. Por ejemplo, se ha propuesto el punto de corte de 21 en diferentes poblaciones de Colombia ${ }^{17}$, Chi$\mathrm{le}^{18}$, Portugal $^{19}$ y Japón ${ }^{20}$.

La escolaridad y la edad son dos factores que afectan al rendimiento en los test de cribado. Para compensar el efecto de la escolaridad en el MMSE se han propuesto diferentes estrategias, como agregar puntos a las personas con baja escolaridad, eliminar o modificar reactivos, indicar diferentes puntos de corte, las cuales han presentado limitaciones para detectar adecuadamente probable DCL ${ }^{21}$. En el MoCA se ha propuesto agregar 3 o $4^{22}$ o 2 puntos para personas entre 8 a 12 ańos $^{18}$.

Otra alternativa es considerar criterios diagnósticos. En diferentes clasificaciones se considera que no presente demencia, sin problemas en actividades de la vida diaria y una desviación estándar por debajo de la media en test $\operatorname{cognitivos}^{23}$. En ente sentido, en nuestro estudio utilizando el MoCA, el punto de corte para indicar probable DCL es menos de 10 para las personas con 75 o más ańos sin escolaridad y en 20 para las personas menores de 75 años con 10 o más años de escolaridad. También se pueden utilizar percentiles; por ejemplo, en nuestro estudio utilizando el percentil 25 se encuentra el $20 \%$ de las personas con DCL.

El presente estudio se realizó en una muestra con un rango amplio de edad y escolaridad; sin embargo, una limitación fue el tamaño de la muestra, por lo que sería recomendable aumentarla en futuros estudios. También, utilizar una evaluación mediante indicadores más sensibles al DCL, para identificar la sensibilidad, especificidad y valor predictivo en la comunidad.

\section{- CONCLUSIONES}

El punto de corte para determinar probable deterioro cognitivo en población clínica en el MoCA se ha encontrado en 26; este valor usado en la comunidad sobreestima la proporción de personas con dicho padecimiento. Por otro lado, con el MMSE da una proporción parecida a lo reportado en la literatura. La sobreestimación puede generar repercusiones en las políticas públicas, al emitir un diagnóstico clínico y en la investigación. Las puntuaciones obtenidas con el MMSE y el MoCA tienen relación entre ellas; sin embargo, las puntuaciones en el MoCA son menores y presentan una mayor dispersión en grupos de diferente edad y escolaridad, por lo que podría utilizar un punto de corte diferente para determinar DCL en población en la comunidad

\section{Conflicto de intereses}

Los autores declaran no tener ningún conflicto de intereses.

\section{Fuente de financiación}

La presente investigación no ha recibido ayudas específicas provenientes de agencias del sector público, sector comercial o entidades sin ánimo de lucro. 


\section{BIBLIOGRAFÍA}

1. Lin JS, O'Connor E, Rossom RC, Perdue LA, Eckstrom E. Screening for Cognitive Impairment in Older Adults: A Systematic Review for the U. S. Preventive Services Task Force. Ann Intern Med. 2013;159(9):60112. doi: 10.7326/0003-4819-159-9-201311050-00730

2. Wu Y-T, Fratiglioni L, Matthews FE, Lobo A, Breteler MMB, Skoog I, et al. Dementia in western Europe: epidemiological evidence and implications for policy making. Lancet Neurol. 2016;15(1):116-24 doi: 10.1016/S1474-4422(15)00092-7

3. Yokomizo JE, Simon SS, de Campos Bottino CM. Cognitive screening for dementia in primary care: a systematic review. Int Psychogeriatrics. 2014;26(11):1783-804. doi: 10.1017/ S1041610214001082

4. Deckers K van Boxtel MPJ, Schiepers OJG, de Vugt M, Muñoz Sánchez JL, Anstey KJ, et al. Target risk factors for dementia prevention: a systematic review and Delphi consensus study on the evidence from observational studies. Int J Geriatr Psychiatry. 2015:30(3):234-46. doi:10.1002/gps.4245

5. Mitchell AJ. A meta-analysis of the accuracy of the mini-mental state examination in the detection of dementia and mild cognitive impairment. J Psychiatr Res. 2009:43(4):411-31. doi: S002239560800112X

6. Nasreddine ZS, Phillips NA, Bédirian V, Charbonneau S, Whitehead V, Collin I, et al. The Montreal Cognitive Assessment, MoCA A Brief Screening Tool For Mild Cognitive Impairment. J Am Geriatr Soc. 2005;53(4):695-9. doi: 10.1111/j.1532-5415.2005.53221.x

7. Roalf DR, Moberg PJ, Xie SX, Wolk DA, Moelter ST, Arnold SE. Comparative accuracies of two common screening instruments for classification of Alzheimer's disease, mild cognitive impairment, and healthy aging. Alzheimer's Dement. 2013;9(5):529-37. doi:S1552526012024636

8. van Steenoven I, Aarsland D, Hurtig H, Chen-Plotkin A, Duda JE, Rick J, et al. Conversion between Mini-Mental State Examination,
Montreal Cognitive Assessment, and Dementia Rating Scale-2 scores in Parkinson's disease. Mov Disord. 2014:29(14):1809-15. doi: $10.1002 / \mathrm{mds} .26062$

9. Nilsson FM. Mini Mental State Examination (MMSE)? probably one of the most cited papers in health science. Acta Psychiat Scand. 2007:116(2):156-7. doi: 10.1111/j.1600-0447.2007.01037.x

10. Davis DHJ, Creavin ST, Yip JLY, Noel-Storr AH, Brayne C, Cullum S Montreal Cognitive Assessment for the diagnosis of Alzheimer's disease and other dementias. Cochrane Database Syst Rev. 2015:10:CD010775 doi: 10.1002/14651858.CD010775.pub2.

11. Aguilar-Navarro SG, Mimenza-Alvarado AJ, Palacios-García AA Samudio-Cruz A, Gutiérrez-Gutiérre LA, Ávila-Funes JA. Validez y confiabilidad del MoCA (Montreal Cognitive Assessment) para el tamizaje del deterioro cognoscitivo en México. Revista Colombiana de Psiquiatría. 2018;47(4):237-243. doi: 10.1016/j. rcp.2017.05.003

12. Gutiérrez JR, Guzmán GG. Definition and prevalence of mild cognitive impairment. Rev Esp Geriatr Gerontol. 2017;52(Suppl-1):3-6 doi: 10.1016/S0211-139X(18)30072-6

13. Arronte-Rosales A, Beltrán-Castillo N Correa-Muñoz E, Mart'nez-Maldonado ML, Mendoza-Núñez VM. Manual para la evaluación gerontológica integral en la comunidad.México: Facultad de Estudios Superiores Zaragoza; 2008

14. Chambers IW, Sivananthan S, Brayne C. Is Dementia Screening of Apparently Healthy Individuals Justified? Adv Prev Med. 2017;2017:9708413. doi: 10.1155/2017/9708413

15. Pandya SY, Clem MA, Silva LM, Woon FL. Does mild cognitive impairment always lead to dementia? A review. J Neurol Sci. 2016;369:57-62. doi: S0022510X16304683.

16. Apostolo J Holland C O'Connell MDL Feeney J, Tabares-Seisdedos R, Tadros G, et al. Mild Cognitive Decline. A Position State- ment of the Cognitive Decline Group of the European Innovation Partnership for Active and Healthy Ageing (EIPAHA). Maturitas. 2016:83:83-93. doi: 10.1016/.maturitas.2015.10.008

17. Gómez F, Zunzunegui M, Lord C, Alvarado B, García A. Applicability of the MoCA-S test in populations with little education in Colombia. Int J Geriatr Psychiatry. 2013;28(8):813-20. doi:10.1002/ gps.3885

18. Delgado C, Araneda A, Behrens MI. Validación del instrumento Montreal Cognitive Assessment en español en adultos mayores de 60 años. Neurología. 2019:34(6):376-85. doi: S0213485317301020

19. Gallego ML, Ferrándiz MH, Garriga OT, Nierga IP, López-Pousa S Franch JV. Validación del Montreal Cognitive Assessment (MoCA) test de cribado para el deterioro cognitivo leve. Datos preliminares. Alzheimer Realidades e Investigación en Demencia. 43:4-11.

20. Narazaki K, Nofuji Y, Honda T, Matsuo E, Yonemoto K, Kumagai S Normative Data for the Montreal Cognitive Assessment in a Japanese Community-Dwelling Older Population. Neuroepidemiology. 2013;40(1):23-9. doi: 10.1159/000339753

21. Franco-Marina F, García-González JJ, Wagner-Echeagaray F, Gallo J, Ugalde 0, Sánchez-García S, et al. The Mini-mental State Examination revisited: ceiling and floor effects after score adjustment for educational level in an aging Mexican population. Int Psychogeriatrics. 2010:22(1):72-81. doi:\$1041610209990822

22. Zhou Y, Ortiz F, Nuñez C, Elashoff D, Woo E, Apostolova LG, et al. Use of the MoCA in Detecting Early Alzheimer's Disease in a Spanish-Speaking Population with Varied Levels of Education. Dement Geriatr Cogn Dis Extra. 2015;5(1):85-95. doi: 10.1159/000365506

23. Bradfield NI, Ames D. Mild cognitive impairment: narrative review of taxonomies and systematic review of their prediction of incident Alzheimer's disease dementia. BJ Psych Bulletin. 2019:14:1-8. doi: 10.1192/bjb.2019.77 